\title{
Transposition
}

Musique et Sciences Sociales

$8 \mid 2019$

Musique : patrimoine immatériel ?

\section{« Le patrimoine c'est nous! » Voix plurielles autour du cante alentejano}

"We are the Heritage!" Plural Voices on Cante Alentejano

\section{Maria do Rosário Pestana et Maria José Barriga}

\section{(2) OpenEdition}

Journals

Édition électronique

URL : http://journals.openedition.org/transposition/3353

DOI : 10.4000/transposition.3353

ISSN : 2110-6134

Éditeur

CRAL - Centre de recherche sur les arts et le langage

\section{Référence électronique}

Maria do Rosário Pestana et Maria José Barriga, « « Le patrimoine c'est nous ! » Voix plurielles autour du cante alentejano », Transposition [En ligne], 8 | 2019, mis en ligne le 15 septembre 2019, consulté le 17 décembre 2020. URL : http://journals.openedition.org/transposition/3353 ; DOI : https://doi.org/ 10.4000/transposition.3353

Ce document a été généré automatiquement le 17 décembre 2020.

La revue Transposition est mise à disposition selon les termes de la Licence Creative Commons Attribution - Partage dans les Mêmes Conditions 4.0 International. 


\section{« Le patrimoine c'est nous! » Voix plurielles autour du cante alentejano}

"We are the Heritage!" Plural Voices on Cante Alentejano

Maria do Rosário Pestana et Maria José Barriga

\section{Introduction'}

J'ai pris goût au cante [alentejano] avec mon père, à Serpa, en [écoutant les chanteurs à] la taverne en face de chez nous... j'en avais la chair de poule... Cette chose merveilleuse qui est la leur... moi, ça m’a remuée. Mon fils aîné a vingt-cinq ans et il n'a pas grandi avec ce programme d'enseignement du cante alentejano dans les écoles. Mais... il a une mère qui aime énormément chanter, et à chaque fois que je voyageais... je chantais tout le temps, et lui, il me

faisait taire : « Oh là, là maman! que c'est ringard ! c'est nul! »

Avec mon fils de vingt-et-un ans, c'est la même rengaine : «Oh maman, taisez-vous! Encore ces

chansons..."

Par contre, celui-ci [référence à son troisième fils, le plus jeune], il a bénéficié de ce programme. Il a eu quatre ans de cante [alentejano] à l'école. Il chante avec ses camarades. Cela fait que mon fils me demande : «Maman, c'était comment déjà cette chanson que vous chantiez? " Juste pour me faire chanter... car une fois qu'il retrouve ses camarades, il ne s'en souvient plus. Donc, cela 
exerce une grande influence. Les parents, les ensembles, l'école, le sentiment d'appartenance, au fond, c'est ce que vous [elle se tourne vers les chanteurs de la table-ronde, à ses côtés] aimez tous lorsque vous allez chanter dans les ensembles. C'est appartenir à un groupe. C'est l'identité : la rencontre avec l'autre. Et ça, c'est une chose merveilleuse que le cante alentejano nous fait sentir ${ }^{2}$. (Teresa Almeida, Centre UNESCO de Beja, 2018)

$1 \quad$ Notre étude analyse la transformation symbolique du cante alentejano (Alentejo/sud du Portugal) et les attentes des détenteurs de la tradition et des agents culturels après son inscription sur la liste du Patrimoine Culturel Immatériel de l'Humanité de l'UNESCO, le 27 novembre 2014. Dans la citation ci-dessus, extraite d'une rencontre ouverte au public intitulée «Le patrimoine, c'est nous ${ }^{3}$ !», Teresa Almeida, originaire de Serpa, relève les effets de cette transformation symbolique chez son plus jeune fils, jeune alentejano urbain, lui ayant permis de s'identifier au cante alentejano. Cette expérience couvre les quatre dernières années de la famille de Teresa; elle fait allusion au programme d'enseignement du cante à l'école, une des mesures mises en œuvre lors du processus de candidature de cette pratique vocale à la Liste Représentative du Patrimoine Culturel Immatériel de l'Humanité de l'UNESCO ${ }^{4}$.

2 Nos principales questions ont été les suivantes: comment les protagonistes du cante alentejano conçoivent-ils ce nouveau statut? De quelle façon des ensembles vocaux constitués depuis des décennies à la faveur de processus « d'objectivation culturelle » envisagent-ils ces transformations? Dans quelle mesure la reconnaissance par l'UNESCO constitue-t-elle une opportunité de dialogue entre détenteurs des traditions, groupes établis, musiciens et agents culturels, et comment affecte-t-elle les pratiques musicales locales?

3 Ces questions deviennent pertinentes dans le contexte de la littérature récente sur le patrimoine culturel. En particulier, elles s'inscrivent dans les approches déconstructionnistes des "opérations métaculturelles qui transmettent aux personnes vivantes les valeurs et les méthodes muséologiques ${ }^{5}$ ». On les relève aussi dans les études qui soulignent l'importance de la valorisation des "détenteurs" et des «transmetteurs » de traditions, ainsi que de « leur habitus et [de] leur habitat [...], afin de préserver l'ensemble du système en tant qu'entité vivante ${ }^{6} »$. Nous analyserons ce processus « post-inscription sur la liste de l'UNESCO » selon les tensions créées autour de polarités: apprentissage scolaire vs pratique au sein des ensembles locaux; chanteurs plus âgés vs jeune génération; ensembles traditionnels vs projets artistiques contemporains; politiques culturelles locales $v s$ attentes et besoins des ensembles.

4 Nous partons de l'hypothèse que le patrimoine immatériel se tisse et se transmet à travers des situations de rencontre et d'interaction, processus centrés sur des personnes qui partagent un même socle de connaissances ${ }^{7}$. De notre point de vue, le cante alentejano a acquis une nouvelle importance dans la vie des personnes concernées, ce qui mérite d'initier un débat et une réflexion critique, afin de faire apparaître les différentes perceptions des acteurs ayant vécu le processus d'inscription sur la liste de l'UNESCO. Notre étude est fondée sur un travail de terrain effectué dans différentes localités, en accompagnant des individus et des ensembles. Elle présente différents 
points de vue sur les questions formulées, dans le but de parvenir à une compréhension plurielle, pouvant permettre à ceux qui sont objets de l'enquête d'en tirer profit ${ }^{8}$. Notre étude que nous avons menée vise non seulement à contribuer à la connaissance du cante alentejano dans les années suivant l'inscription sur la liste de l'UNESCO, mais également à initier une discussion interne plus large et transversale entre les différents acteurs impliqués dans cette pratique culturelle.

Comme méthodologie de travail de terrain, nous avons mené des entretiens informels et avons interrogé des dizaines de personnes liées au cante. Il nous a été dit, à plusieurs reprises, qu'il serait nécessaire d'organiser des réunions publiques pour aborder les interrogations communes aux différents protagonistes du cante. Nous avons donc étendu notre méthodologie, centrée au départ sur ces entretiens, à la réalisation d'événements publics. Pour ces derniers, nous avons invité chanteurs, mestres, paroliers, compositeurs, agents culturels locaux et régionaux et personnes étudiant le cante. La première rencontre s'est tenue en 2015, à Reguengos de Monsaraz, dans le cadre du colloque Práticas musicais no Alentejo: a terra, as memórias e o património [Pratiques musicales en Alentejo: terre, mémoires et patrimoine]. Les participations orales à cette rencontre ont été transcrites et publiées en $2017^{9}$. Trois ans plus tard, nous avons organisé des séances de débat intitulées «Le patrimoine, c'est nous!» au Centre Unesco de Beja, à la Casa do Povo de S. Martinho das Amoreiras (commune d'Odemira), et à la Casa do Cante de Serpa.

6 Considérant que les acteurs culturels font du patrimoine immatériel un élément actif de transmission culturelle et de l'identité collective, nous avons tracé le cadre historique de cette pratique musicale au $\mathrm{XX}^{\mathrm{e}}$ siècle, en nous appuyant sur la littérature existante et nos propres recherches d'archives. Notre étude explore à la fois ces dimensions historiques et synchroniques.

7 Les deux auteures ont une relation particulière avec le cante alentejano: Maria José Barriga a appris le cante en contexte familial et a observé différentes pratiques musicales dans l'Alentejo comme ethnologue ${ }^{10}$; Maria do Rosário Pestana a étudié la première collection d'enregistrements sonores non commerciaux du cante alentejano (1939-40), coordonné deux études approfondies sur le territoire portugais auprès de groupes de cante alentejano et effectué plusieurs enquêtes de terrain ${ }^{11}$.

\section{Le cante alentejano et la région de l'Alentejo}

8 Le cante ${ }^{12}$ ou cante alentejano, également appelé cantar à Alentejana (littéralement « chanter à la mode de l'Alentejo ») est une pratique vocale polyphonique, la plupart du temps a cappella, chantée dans des lieux publics ou privés, par des ensembles formellement institués (comprenant entre quinze et trente membres, vêtus très souvent de costumes traditionnels et un chef de chœur ou mestre) ou par des ensembles informels, mixtes, d'hommes ou de femmes. Il y prédomine trois rôles principaux - le ponto (soliste) qui initie le chant ; l'alto (soliste) qui entre après le ponto et chante une tierce au-dessus, entonnant parfois la quinte ou la dixième et ornant parfois la mélodie ; et les baixos ou segundas, qui chantent à l'unisson et interviennent après l'alto.

9 Dans le dossier de candidature, on peut lire que «le ponto et l'alto jouent un rôle prépondérant dans le cante. L'un des deux, ou, à défaut, tout autre détenteur de la tradition, prend la tête de l'ensemble, forme les chanteurs, sélectionne et transmet le répertoire, et dirige les répétitions et les présentations. Ce rôle de mestre ou ensaiador 
[ « chef de chœur »] a récemment été formalisé, en étant reconnu publiquement et en recevant le titre de mestre ${ }^{13} »$. Les pièces du répertoire de cante alentejano sont strophiques, chaque nouvelle strophe étant initiée par le ponto. La mélodie se nomme estilo. Le quatrain introductif est appelé cantiga tandis que les strophes qui suivent la cantiga sont appelées moda. La même cantiga peut être utilisée pour introduire différentes modas et le thème de la cantiga peut ne pas correspondre à la moda qui suit. Le titre d'une moda est lié au texte de son premier vers ${ }^{14}$. Voici les paroles de la moda intitulée «Que inveja tens tu das rosas» (« Pourquoi es-tu jalouse des roses») :

\begin{tabular}{|l|l|}
\hline Cantiga & Cantiga \\
Sous la nuit, dans les landes, & Na noite, pelas campinas \\
Le soleil court après la lune & Anda o sol atrás da lua \\
Ainsi va mon destin, & Assim é a minha sina \\
Ma bien aimée, après le tien. & Meu amor, atrás da tua. \\
Moda & Moda \\
Pourquoi es-tu jalouse des roses, & Que inveja tens tu das rosas \\
Quand comme elles, tu es si belle, & Se és linda como elas são \\
Reine des fleurs & A rainha das flores \\
Chantées par tes mains & Cantadas por tuas mãos \\
{$[. .]$.} & {$[. .]$.} \\
\hline
\end{tabular}

Ensemble de femmes Moças da Aldeia de Sta.Clara de Louredo, Beja, 26 mai 2018, 3e Rencontre de ensembles vocaux à Alvide, Cascais.

10 Alors que la création de nouveaux textes poétiques suit le courant de l'actualité en abordant des thèmes liés à des événements et préoccupations actuelles, la création de nouveaux estilos (terme utilisé pour faire référence aux mélodies) est plus rare. À la fin $\mathrm{du} \mathrm{XX}^{\mathrm{e}}$ siècle, l'ethnomusicologue Salwa Castelo-Branco signalait le fait que, bien qu'aucun recueil de chansons ne mentionne les noms des paroliers et compositeurs, ceux-ci étaient très connus, admirés localement; elle donne l'exemple du poète populaire Manuel Castro et du chanteur António Luís Fialho, auquel l'on attribue la création de « dizaines de estilos ${ }^{15} »$.

11 Le cante alentejano est une pratique développée en Alentejo, une vaste région à faible densité démographique $(33 \%$ du territoire national et seulement $7,6 \%$ de la population) ${ }^{16}$. Elle se trouve au centre-sud du Portugal continental et est délimitée par l'océan atlantique à l'ouest, l'Espagne à l'est et la région de l'Algarve au sud. Selon le $15^{\mathrm{e}}$ recensement général de la population (2011), la région est constituée par cinq sousrégions (NUTS III). Le taux d'analphabétisme y atteint 9,57\% ; la part de la population de plus de 65 ans est de $26,16 \%$, et les ressortissants étrangers représentent $3,12 \%{ }^{17}$. Ces dernières décennies, le vaste territoire de l'Alentejo a subi de profonds changements. Après des années successives d'exode migratoire dû à la précarité du travail rural salarié, après la révolution de 1974 qui a réinstauré la démocratie au Portugal, les travailleurs agricoles ont déclenché un mouvement d'occupation des terres et se sont organisés en coopératives de production, conduisant ainsi la Réforme Agraire $^{18}$. L'adhésion à l'Union européenne en 1986 a imprimé de nouveaux changements dans la gestion et l'utilisation du territoire. Ces dernières années, cette région est devenue également un espace de tourisme et de consommation du paysage et du patrimoine ${ }^{19}$. 


\section{Le processus d'institutionnalisation du cante alentejano}

12 La vaste littérature produite tout au long $\mathrm{du} \mathrm{XX}^{\mathrm{e}}$ siècle montre que le cante alentejano est enraciné dans la région depuis des décennies. Tout au long du XXe siècle, il a été marqué par des processus successifs d'« objectivation culturelle » (avec la sélection, la décontextualisation et l'attribution de nouvelles intentions et significations au cante alentejano $)^{20}$, initialement $\mathrm{du}$ fait d'initiatives individuelles venant de folkloristes et d'ethnographes, puis, pendant la période autocratique de la dictature militaire (1926-32) et du régime totalitaire de l'Estado Novo (1933-74), dans le cadre d'une stratégie politique qui prétendait contrôler la vie sociale des Portugais sous une identité "unifiée». L'institutionnalisation des ensembles masculins a été l'une des facettes visibles de cette transformation. Dans les années 1920-1930, une nouvelle formalisation des représentations de ces ensembles masculins, vêtus de costumes traditionnels et portant les instruments représentatifs de leur travail et de la sociabilité du monde rural, reconfigure cette pratique. Pendant le régime autocratique, le cante est intégré à des événements de promotion de la région : en 1937 a lieu, dans la ville de Lisbonne, la première présentation publique d'ensembles vocaux de l'Alentejo. Parmi les ensembles constitués pendant cette période, il en est un qui est toujours en activité en 2018 : le Groupe choral des Mineurs d'Aljustrel, fondé en 1926.

Dans les années trente, différentes institutions de l'Estado Novo ont développé des actions de contrôle: (i) la constitution formelle des ensembles, avec leur intégration dans les Casas do Povo (Maisons du Peuple), placées sous la tutelle du régime dictatorial, et l'organisation de concours et d'événements publics; (ii) l'interdiction d'ensembles constitués spontanément sans autorisation des autorités locales; (iii) la prohibition des réunions après vingt-et-une heures ${ }^{21}$ (les chanteurs qui se réunissaient après cette heure-là faisaient l'objet de procès-verbaux qui pouvaient les mener en prison). Ces actions de propagande du régime ont imposé l'idée que le cante était masculin, en excluant presque toujours les femmes des ensembles formellement institués ${ }^{22}$. C'est encore pendant cette période que le cante alentejano a été défini comme la principale pratique de l'identité musicale de l'Alentejo, au mépris d'autres pratiques locales comme le cante ao Baldão, les Balhos, ou la Moda Campaniça ${ }^{23}$. De cette période datent aussi les premiers enregistrements sonores non commerciaux. En 1939-1940, le folkloriste Armando Leça (1891-1977) enregistre des modas où les rôles de "ponto", « alto » et « baixos » sont pris en charge par des hommes et des femmes ${ }^{24}$. Vingt-cinq ans plus tard, l'ethnographe corse Michel Giacometti édite le LP Alentejo, intégré dans la collection Antologia da Música Regional Portuguesa [Anthologie de la Musique Régionale Portugaise], qui sera suivi des Cantos Tradicionais do Distrito de Évora [Chants Traditionnels du District d'Évora] en 1965, des Bailes Populares Alentejanos [Bals Populaires de l'Alentejo] en 1968, des Bonecos de Santo Aleixo [Marionnettes de Santo Aleixo], également en 1968, et de Alentejo, Música Vocal e Instrumental [Alentejo, Musique Vocale et Instrumentale] en $1974^{25}$.

Après la restauration de la démocratie en 1974, des ensembles de cante alentejano, des mouvements associatifs, le pouvoir local et d'autres agents ont resignifié le cante alentejano en mettant notamment l'accent sur l'importance des mémoires de l'oppression et de la résistance de ses protagonistes durant le régime fasciste. Les 
principaux discours ont gravité une fois encore autour du passé et de l'identité. Cette période a vu émerger de nouveaux ensembles vocaux dans la région ${ }^{26}$, mais aussi dans l'aire métropolitaine de Lisbonne, où de nombreux alentejanos ont migré. Dans cette région de la capitale, sont également apparus des ensembles féminins formellement constitués :

Le nombre d'ensembles masculins a toujours été supérieur à celui des ensembles féminins. Cependant, la formation d'ensembles féminins croît depuis les années 1980. Le renforcement de la présence des femmes dans les ensembles impose « une vision holistique du patrimoine culturel immatériel, et une nouvelle compréhension de la mémoire collective future $»^{27}$.

Il faut souligner les initiatives prises pour transmettre et sauvegarder le cante alentejano. On peut repérer, dans les années 1980, des actions ponctuelles de transmission du cante auprès des plus jeunes afin de contrecarrer le vieillissement des ensembles. La constitution d'ensembles d'enfants et/ou d'adolescents a été la stratégie adoptée. Il faut noter la création à Castro Verde, en 1987, de l'ensemble choral d'enfants et adolescents «Os Carapinhas ", fondé par l'association Cortiçol. Certains de ses éléments ont intégré par la suite des ensembles locaux d'adultes.

Divers projets d'enseignement du cante dans des localités de l'Alentejo, et même dans l'aire métropolitaine de Lisbonne, ont suivi ces premières tentatives. Comme l'indique Salwa Castelo-Branco, « les municipalités ont remplacé le gouvernement en donnant des appuis financiers et logistiques (espaces pour les répétitions, transport, etc.) ${ }^{28}$ ». Parallèlement à ces initiatives institutionnelles, on constate des dynamiques générées dans les ensembles par l'organisation d'événements locaux comme des rencontres, festivals, fêtes d'anniversaire. On a pu observer le même phénomène pour d'autres ensembles folkloriques un peu partout dans le pays ${ }^{29}$. Les ensembles ont tissé des réseaux de relations entre eux, ce qui leur permet d'organiser des rencontres et des festivals sous la forme d'échanges (l'ensemble qui organise l'événement prend à sa charge les frais de logement et d'alimentation des ensembles invités et s'engage à participer à leurs événements, sans demander de cachet). De ce fait, les ensembles parcourent l'Alentejo et se rendent dans les différentes localités de migration des alentejanos de l'aire métropolitaine de Lisbonne et même en dehors du Portugal. Paraphrasant Benedict Anderson, Salwa Castelo-Branco affirme que ces rencontres ont permis de créer une « communauté imaginée » à travers l'expression sonore ${ }^{30}$.

D'autres types d'actions ont en outre été menés pour sauvegarder, promouvoir et divulguer le cante alentejano par des associations protectrices du patrimoine local, parmi lesquelles Cortiçol, Moda-Associação do Cante Alentejano et la confrérie Confraria do Cante.

18 C'est à partir de 2014 que les projets cherchant à dynamiser cette pratique se sont réellement intensifiés. Certains avaient pour but de promouvoir le rajeunissement du cante : dès 2014, l'événement As Novas Vozes do Cante [Les nouvelles voix du cante] lors de la Festa do Avante ! ; l'année suivante, les $1^{\text {res }}$ rencontres des ensembles vocaux de jeunes I Encontro de Grupos Corais Juvenis à Reguengos de Monsaraz, entre autres. Les représentations croisant le cante avec d'autres formes d'expression ou genres musicaux se sont multipliées. 


\section{La candidature à la liste du Patrimoine Culturel de I'UNESCO et le début du processus post-inscription}

19 La proclamation de l'inscription du cante alentejano sur la liste du Patrimoine Culturel Immatériel de l'Humanité de l'UNESCO a eu lieu le 27 novembre 2014. La municipalité de la ville de Serpa a assuré la promotion de la candidature; l'instruction et la coordination scientifiques du projet ont été réalisées par les anthropologues Paulo Lima et Jorge Freitas Branco, ainsi que par l'ethnomusicologue Salwa Castelo-Branco. L'objectif principal de cette candidature était d'organiser et de consolider un mouvement associatif local visant à la sauvegarde de cette pratique de chant polyphonique originaire de la région de l'Alentejo. La mairie de Serpa réussit à cette époque à rassembler cinquante-sept ensembles de cante alentejano pour soutenir le dossier de la candidature ${ }^{31}$. Ana Paula Amendoeira, directrice régionale de la culture de l'Alentejo, a souligné le fait que le processus de candidature n'avait pas été suffisamment inclusif, n'avait pas permis une implication satisfaisante de tous les chanteurs et ensembles, et qu'il était nécessaire de "promouvoir des rencontres élargies avec tous les intéressés " pour divulguer notamment le «projet de candidature ${ }^{32}$ ». En 2017, soit trois ans après l'inscription, des chanteurs et chefs de chœur appelaient encore à être informés du plan de sauvegarde mis en œuvre consécutivement à l'inscription sur la liste de l'UNESCO.

Cette inscription a, depuis, selon Castelo-Branco,

éveillé l'intérêt des jeunes pour ce mode d'expression, ce qui a suscité l'émergence d'ensembles exclusivement constitués de jeunes, leur intégration dans des ensembles déjà existant, l'apparition de petites formations composées de chanteurs s'accompagnant à la viola campaniça [guitare d'environ $94 \mathrm{~cm}$ avec 10 cordes traditionnelle de l'Alentejo] et la configuration de nouveaux langages musicaux mêlant le cante à d'autres styles de musique populaire ${ }^{33}$.

21 Tout au long de ce processus, le cante a acquis une place de plus en plus centrale dans les projets artistiques de jeunes musiciens (qui sont de plus en plus visibles dans les médias) et dans les politiques culturelles des municipalités. L'étude que nous avons réalisée a révélé aussi une augmentation du nombre d'ensembles musicaux après la reconnaissance du cante par l'UNESCO en 2014. Si, en 2013, nous avions enregistré 158 groupes de cante alentejano $0^{34}$, en 2018 , dans un nouveau recensement, également coordonné par l'une des auteurs de cet article, on en identifiait 177.

Parmi les milieux amateurs, ont surgi des chanteurs professionnels jouissant d'une grande visibilité, y compris dans le cadre des industries du cinéma et de la culture. Selon l'anthropologue Susana Mareco, «le principal aspect qui différencie ces artistes des ensembles vocaux (aussi bien de la nouvelle génération que de l'ancienne) [...] est que la musique est leur activité professionnelle, et qu'ils sont en concurrence pour ce qui est des publics, des maisons d'édition, des radios etc..$^{35}$ ». L'un des protagonistes de ce nouveau cadre de professionnalisation est Pedro Mestre, chanteur, chef de cœur, compositeur, producteur et instrumentiste de viola campaniça. Il jouit depuis dix ans d'une forte médiatisation, étant aussi responsable de l'enseignement formel du cante à l'école. Il est devenu un modèle pour les jeunes chanteurs qui tentent de réussir à construire une vie professionnelle basée sur la pratique de ce chant polyphonique. Les contextes de présentation se diversifient, chaque nouveau protagoniste construit une 
nouvelle façon de performer le chant et d'adapter les caractéristiques traditionnelles à son projet individuel.

Parallèlement, la participation des femmes s'est intensifiée, dans la constitution des ensembles formels comme dans l'organisation d'événements. En 2016 le mouvement démocratique des femmes (Movimento Democrático de Mulheres) a recensé soixante et un ensembles de cante alentejano féminins, et a promu diverses rencontres afin de valoriser leur participation et susciter une réflexion sur leur rôle en tant que chanteuses, parolières, mestres ou organisatrices d'événements ${ }^{36}$.

La réactivation d'ensembles formés dans la première moitié du $\mathrm{XX}^{\mathrm{e}}$ siècle, mais qui avaient disparu depuis plusieurs années, date également de l'époque de la candidature. Des événements où la promotion touristique est liée à la divulgation du cante Alentejano ou à celle de produits régionaux se sont multipliés depuis son inscription sur la liste de l'UNESCO : CanteFest' 15 à Serpa; hommage au Cante à Amareleja; la fête du Cante dans les Terres du Grand Lac à Reguengos de Monsaraz en 2015; Ovibeja, événement de promotion de l'agriculture et de la production animalière à Beja, qui a réuni en 2015 plus de 2000 chanteurs lors de la $1^{\text {re }}$ Grande Rencontre du Cante. Ces dynamiques résultent d'initiatives plurielles, c'est-à-dire de divers acteurs aux intérêts différents.

\section{« Le patrimoine, c'est nous ! » : rencontres avec des chanteurs, mestres et agents culturels}

Les rencontres publiques que nous avons organisées portaient toutes sur le cante alentejano en contexte "post-inscription sur la liste de l'UNESCO »: la conduite du processus de candidature, les politiques culturelles et économiques en cours, les dynamiques des ensembles formellement institués, le rôle de chacun dans la sauvegarde du cante et les attentes par rapport à l'avenir.

Le point de départ de nos discussions était la sauvegarde du cante alentejano, mais les participants ont rapidement élargi le débat pour aborder d'autres sujets, comme l'enseignement formel du cante dans les écoles publiques, le profil du mestre, les «dangers " de la standardisation par l'effacement des variantes locales, les modes de transmission informels, la participation des femmes, les contextes performatifs (spectacles, représentations), le processus de patrimonialisation lui-même, la communauté, ou encore l'ensemble qui avait été choisi pour représenter le cante alentejano à l'UNESCO, à Paris. Nous allons analyser ce processus au prisme des quatre polarités suivantes: apprentissage scolaire $v s$ pratique au sein des ensembles locaux; chanteurs plus âgés vs nouvelle génération; ensembles traditionnels vs projets artistiques contemporains; politiques culturelles locales vs attentes et besoins des ensembles.

\section{Apprentissage à l'école vs pratique au sein des ensembles locaux}

27 Un sujet très discuté aussi bien lors des entretiens qu'au cours des rencontres publiques a été celui de l'enseignement formel du cante alentejano à l'école. Celui-ci a été présenté par les responsables municipaux comme un investissement dans la transmission de la connaissance nécessaire au maintien de la pratique. Odete Borralho, adjointe à la culture de la mairie de Serpa, présentait le programme d'enseignement mis en place en 2008 dans les écoles publiques au niveau élémentaire (enfants âgés de 6 à 10 ans) - dans 
lequel a été intégré le cante grâce à un projet homologué par le ministère de l'éducation - de la façon suivante :

Dix ans après, notre bilan c'est que nous aimerions que les enfants qui veulent continuer à apprendre, mais qui sont encore trop jeunes pour faire partie d'un ensemble, puissent continuer à apprendre. Nous pensons offrir ici, dans la Casa do Cante, des cours le samedi, avec l'aide des ensembles vocaux [...]. Il faut que quelque chose d'autre se fasse [en dehors de l'enseignement du cante dans le premier cycle] et pour que cela surgisse, il est fondamental que ce soit vous [elle s'adresse aux chanteurs qui se trouvent dans l'auditoire] qui transmettiez cette connaissance. Je sais en effet que certaines critiques disent: «ce qui se fait dans les écoles avec le cante est partout la même chose ». Parce que nous à Serpa nous avons le Cantar de Pias, celui de Vila Nova... Chaque village, chaque ensemble a ses caractéristiques. Il est évident que seuls ceux qui intégreront des ensembles réussiront véritablement à apprendre! Mais il est important que les enfants chez qui nous déposons cette graine, et qui veulent la faire grandir, reçoivent ce soutien. Je voudrais obtenir ici, de la part de ces ensembles vocaux, un engagement dans cet effort pour effectuer cette transmission ${ }^{37}$.

Les chanteurs des ensembles les plus anciens, comme José Rocha du groupe Ceifeiros de Cuba, ont évoqué le danger de la standardisation, dans la mesure où un même professeur enseigne dans différentes localités. Ils défendent l'idée selon laquelle l'apprentissage formel à l'école devrait être complété par une pratique informelle au sein des ensembles de chanteurs locaux (Centre Unesco de Beja, 2018). Pour résoudre ce problème, Francisco Baiôa Monteiro, historien et président de l'Associação de Defesa do Património [Association de Défense du Patrimoine], fils de chanteurs, a suggéré que parallèlement à l'apprentissage dans les écoles, les enfants participent à des ensembles de cante locaux. Ainsi, selon lui, le respect de l'identité et des différenciations locales serait assuré. Serafim Silva, chanteur et aussi directeur du Groupe Culturel et Sportif (Grupo Cultural e Desportivo) de Monsaraz, a également mis en garde contre le risque de standardisation. Selon lui, "l'école de Telheiro [doit avoir] un cante, l'école de Reguengos un autre, celle de Portel un autre aussi, celle de Amareleja un autre encore. On ne peut pas tout standardiser, comme les mathématiques ${ }^{38} ! »$. Mais les participants aux rencontres ont reconnu eux-mêmes que l'enseignement du cante dans les écoles a été l'une des actions qui a contribué le plus à revaloriser cette pratique auprès des jeunes des centres urbains - comme le démontre la citation qui ouvre cet article. Au Centre Unesco de Beja, Paulo Colaço, musicien et répétiteur de groupes d'enfants de cante, a souligné l'efficacité de l'enseignement formel du cante dans les écoles et soutenu que cet enseignement devait être transmis oralement.

Diverses initiatives communales d'enseignement formel dans le cadre scolaire ont été identifiées. Pedro Mestre a insisté sur le fait que «certains garçons qui chantent actuellement dans des ensembles vocaux [et qui ont commencé leur apprentissage avec lui, à l'école] ont reçu cette passion qui est la mienne, et c'est cela qu'il est important de transmettre ${ }^{39}$ ». Il a rappelé que, dans les chœurs des ensembles de cante alentejano, certains chanteurs ont été initiés à cette pratique dans le cadre de l'enseignement formel, et qu'on est donc loin du supposé « apprentissage spontané ». Toutefois il a été signalé qu'une grande partie des actuels mestres s'assument comme les détenteurs d'une tradition vécue dans le cadre informel de la famille et/ou de l'espace public. Le chanteur António Caixeiro a insisté sur l'importance de la transmission de la connaissance détenue par ces chanteurs :

Mon idée, ce n'est pas d'aller dans une école et de former trente ou quarante chanteurs. Ce que l'on veut, c'est sensibiliser les enfants à l'existence de ce 
patrimoine, de cette culture, de cette identité qui font partie de notre région. Il est important qu'ils le sachent, qu'ils continuent à le pratiquer ou non. C'est ce qui nous fait agir. Aujourd'hui nous sommes reconnaissants d'avoir appris avec des ensembles qui existaient il y a soixante-dix ou quatre-vingts ans, et nous voulons que dans cinquante ans les plus jeunes puissent aussi dire de nous : ces messieurs chantent depuis cinquante ans. Sans quoi, s'il n'y a pas cette transmission de connaissance vers les plus jeunes, le jour viendra où tout cela se tarira comme une source $^{40}$.

Carlos Arruda, chanteur du Grupo Coral e Etnográfico da Casa do Povo de Serpa, a rappelé que :

Dans les années 1990 [...], quand je chantais dans le cortège, à 13 ans, des groupes d'amis me sifflaient quand je passais en chantant. Le cante était interdit dans les cafés, dans les restaurants [...]. Une phrase courante disait que c'était « ringard» de chanter, car c'était lié à l'alcoolisme. Avec l'UNESCO, on a commencé à parler du cante comme ayant un intérêt culturel [...]. Les mentalités ont changé et de nos jours, là où il était interdit de chanter, on réclame le chant, c'est une réalité différente ${ }^{41}$.

David Cubaixo, chanteur du groupe Rancho de Cantadores da Aldeia Nova de São Bento, a affirmé que, dans le milieu rural où il avait passé sa jeunesse, le cante faisait l'unanimité et était même promu par les érudits locaux. Mais José Soares du groupe Rancho Coral e Etnográfico de Vila Nova de São Bento, a prétendu que «le patrimoine culturel immatériel de l'humanité a apporté un autre statut au cante. Les jeunes ont appris à l'aimer, et les adultes aussi [...]. Avant, la plupart des gens trouvaient cela ringard, nul, et ne l'aimaient $\operatorname{pas}^{42} »$.

Cet intérêt croissant pour le cante explique l'arrivée de jeunes dans les ensembles déjà existants et l'augmentation du nombre d'associations de jeunes formellement instituées, dont certaines sont fortement médiatisées.

\section{Chanteurs plus âgés vs jeune génération}

Nous avons observé, dans les différents discours, une tension latente entre les chanteurs les plus jeunes et les plus âgés. D'une part, les plus âgés revendiquent un statut particulier dans les rôles de solistes du fait d'une autorité et d'une légitimité acquises via leur longue pratique et leur expérience au sein des ensembles de cante. D'autre part, les jeunes réclament ces mêmes rôles de solistes car ils s'estiment suffisamment compétents, et n'aiment pas chanter uniquement dans les baixos, c'est-àdire dans le chœur. Le chanteur Horácio Galego du groupe Rancho Coral Os Camponeses de Vale de Vargo a reconnu que, dans son ensemble, les chanteurs plus âgés n'aimaient pas se présenter à côté des " jeunes gars » et encore moins des enfants. Dans le même temps, les jeunes n'acceptent pas que ce soient les plus âgés qui décident de ce qui est (ou n'est pas) un bon ponto ou un bon alto. La tension est encore plus évidente quand on observe la façon dont les uns et les autres perçoivent leur présentation esthétique sur scène, notamment pour ce qui est des vêtements. Les plus âgés (formés pendant le régime totalitaire et dans un processus d'objectivation culturelle) valorisent des costumes en rapport avec l'ethnographie de la vie rurale d'antan, ce qui parfois déplaît aux plus jeunes qui veulent que les représentations du cante soient en rapport avec le temps présent. Parfois, les plus âgés soulignent la difficulté qu'ont les jeunes à assumer des engagements à l'intérieur de l'ensemble, plaçant souvent leurs intérêts avant ceux du groupe (Horácio Galego, Casa do Cante de Serpa). 


\section{Ensembles traditionnels vs projets artistiques contemporains} propositions artistiques qui apparaissent autour du cante: (1) l'émergence de nouveaux projets artistiques qui voient le cante comme une source de légitimation; (2) les jeunes chanteurs qui, se professionnalisant, se consacrent totalement au cante en en tirant des revenus (en tant que chanteurs, mestres, professeurs, etc.). Le processus de candidature, qui a abouti à la reconnaissance de l'UNESCO, a en effet encouragé l'identification des urbains et des jeunes alentejanos à la pratique musicale du cante et a généré de nouveaux moyens de transmission de connaissances qui ne passent plus par la participation à des groupes de cante officiellement institués et encore moins par la pratique du chant en contexte de travail agricole.

\section{chanteur, soutient que :}

le cante a aujourd'hui une importance qu'il n'avait pas il y a dix ans. À cette époque, quand je suis entré dans le groupe, entrer dans un chœur de "personnes âgées" c'était comme s'arrêter dans le temps, ne pas pouvoir jouer au football, ne pas être avec ses amis, ne pas aller où tous allaient : faire du roller, jouer au foot, au handball et tout le reste. C'était être isolé avec cinquante, vingt, trente hommes qui chantaient à alentejana, c'était, oui, un scandale, qui faisait d'António [son frère] et moi deux gamins rétrogrades [...]. Heureusement, il y a eu la candidature au patrimoine immatériel de l'humanité [...] et nous avons été les pionniers de sa sauvegarde ${ }^{43}$.

Les participants aux rencontres ont fait part de leur inquiétude concernant la valorisation excessive de certains ensembles par rapport à d'autres, responsable, selon eux, du fait que leurs mestres soient bien plus sollicités que les autres pour se produire. Pour José Rocha, du groupe Ceifeiros de Cuba, «l'ensaiador [répétiteur] doit être un membre de l'ensemble, ou quelqu'un qui sait très bien comment chante le groupe " (José Rocha, Centre Unesco de Beja, 2018). Les chanteurs plus âgés s'inquiètent de l'effet que la circulation des ensaiadores pourrait avoir sur le délitement des caractéristiques locales. C'est une question qui a été posée et vivement débattue à Beja par les membres du groupe Ceifeiros de Cuba et des ensembles de Salvada et de Baleizão, ainsi qu'à Odemira par ceux de S. Luís et de Garvão :

Nous [Grupo Coral Alma Alentejana de Garvão] sommes un groupe de la commune de Ourique et on [les instances patrimoniales, la commune] ne fait jamais appel à nous nulle part. Après, le maire vient le jour de la fête [dans la localité siège de l'ensemble communal] et dit : "Il faut préserver le cante alentejano!" Eh bien, moi je demande : comment ${ }^{44}$ ?

Ce sont les ensembles institués depuis plus longtemps, dont les membres sont plus âgés, qui expriment ce sentiment.

Certains groupes soutiennent que depuis 2014 ils ont eu de moins en moins l'occasion de chanter en dehors de leur localité, et que les politiques de sauvegarde du cante ne sont pas discutées par tous. Les chanteurs plus âgés veulent « avoir une voix » dans les politiques de sauvegarde, bénéficier de plus de ressources pour présenter le cante en dehors de leurs localités, et craignent d'être transformés en produits touristiques. 


\section{Politiques culturelles locales vs attentes et besoins des ensembles}

39 Nous avons noté des relations conflictuelles entre ensembles de cante et agents culturels concernant les politiques culturelles de financement des chanteurs locaux et des artistes invités, mais aussi les critères de sélection et les opportunités offertes à la mobilité et la présentation des ensembles. Cependant des chanteurs comme Pedro Mestre ont considéré que l'élaboration par les municipalités de cartes indiquant les lieux de répétition des ensembles ouverts aux touristes relevait des «bonnes pratiques» (selon la formule de l'UNESCO). Deolinda Vaz, adjointe à la culture de la mairie d'Odemira, a présenté comme exemple de cette pratique la Rota Vicentina [Route de la côte vicentine], élaborée par sa commune. Paulo Colaço a également défendu que les ensembles devaient rester dans leur localité :

Une façon d'aider nos villages, avec le tourisme, c'est de ne pas sortir autant et de se produire le plus possible dans sa localité afin de recevoir des personnes extérieures. Le groupe doit représenter son village et, dorénavant, il nous faut repenser cette affaire des sorties. Il est important d'aimer recevoir les amis et les gens d'ailleurs en chantant ${ }^{45}$.

40 Cette stratégie touristique, qui vise à circonscrire davantage l'activité des ensembles aux localités où ils ont leur siège, a été soutenue par les responsables des communes et par les chanteurs professionnels. Cependant, elle n'a pas été aussi bien accueillie par les ensembles et par les chanteurs amateurs, qui pensent que les représentations publiques en dehors des communautés locales (les « sorties ») créent une dynamique positive, qui renforce la participation des membres de l'ensemble aux répétitions.

41 En ce qui concerne la participation des ensembles féminins dans le cante alentejano, les femmes revendiquent pour les ensembles féminins permanents un statut identique à celui des ensembles masculins, arguant du fait que dans le contexte du travail agricole tout le monde chantait :

Parce que nous aussi, nous chantions, hommes et femmes chantaient à cette époque. J'ai 69 ans et j'ai travaillé toute ma vie dans les champs et nous, les femmes, nous chantions quand nous moissonnions, glanions... C'était comme ça, je le sais! [...] L'idée que les femmes ne chantaient pas semble être enracinée dans l'esprit de la plupart des hommes, surtout ici à Serpa. Les femmes ne chantaient peut-être pas dans les tavernes, mais elles chantaient durant les travaux et à la maison ${ }^{46}$ !

Le nombre d'ensembles féminins formellement institués est en augmentation, et ces derniers s'appuient sur des éléments jeunes, comme l'a mentionné Rita Guiomar :

Dans le Grupo Coral Os Ceifeiros de Serpa, nous avons deux jeunes filles, l'une de 10 ans et l'autre de 15 ans et elles ne sont jamais absentes, elles aiment vraiment en faire partie. [...] Leur grand-père chantait dans le groupe de Pias; c'était un bon élément, et elles veulent suivre les traces de leur grand-père. Elles disent même qu'elles aiment que leur grand-père, où qu'il soit, les voient chanter. Elles en sont fières ${ }^{47}$.

Maria Etelvina Rocha, de l'ensemble Coral Madrigal de Vila Nova de São Bento, a soulevé un nouveau problème : la difficulté technique que son ensemble rencontre avec les plateformes digitales, à l'exception de Facebook, qu'elle considère être un moyen de communication très utilisé par elle-même et par les autres chanteuses de l'ensemble pour divulguer leurs activités (Maria Etelvina Rocha, Casa do Cante de Serpa, 2018). Elle demande une médiation personnalisée entre l'information divulguée sur les sites des institutions et l'ensemble. Le problème de la mise en place de plateformes digitales a également été soulevé par Pedro Mestre : 
Ce qu'il faut faire pour que tous les ensembles puissent, en plus du cante, couvrir ce terrain, c'est précisément ce que la Casa do Cante de Serpa a commencé à faire depuis un certain temps, de même que le Centre UNESCO de Beja. Et ce que ces entités ont fait, ce qu'elles font, c'est créer une plateforme, un site, pouvant couvrir tous les ensembles. Le problème c'est que ces institutions demandent aux ensembles de leur faire parvenir des informations et que les ensembles ne réussissent pas à le faire. Ils demandent aux ensembles : mettez à jour vos données sur cette page [internet] ! Mais les ensembles n'en sont pas capables. Il s'agit pour la plupart de travailleurs ruraux. Nous oublions cet aspect. L'UNESCO a accordé le sceau au cante, elle veut défendre le cante amateur, mais après on oublie que ces personnes ne sont pas à même de fournir les réponses. Il faut donc les aider directement. Quand je dis directement, c'est là, avec eux, pendant leurs répétitions ${ }^{48}$.

\section{Conclusion} processus qui en est à ses débuts. Pour cela, nous avons entrepris une étude « avec des personnes", c'est-à-dire que nous avons créé des situations dans lesquelles les protagonistes du cante réfléchissaient avec nous sur le sujet du cante alentejano. Au cours des rencontres publiques que nous avons organisées, les différents protagonistes ont mis en évidence la redéfinition de cette pratique qui s'est opérée ces dernières années : elle est devenue culture et ressource économique. Si le cante alentejano était un phénomène central dans le quotidien rural au milieu du XXe siècle, depuis 2014 il a pris un "second souffle", c'est à dire une nouvelle dynamique sociale et une forte resémantisation.

L'inscription du cante alentejano dans la Liste du Patrimoine Culturel de l'Humanité en a fait un produit culturel. Alors que le territoire de l'Alentejo est devenu un espace de tourisme et de consommation du paysage et du patrimoine, le cante alentejano s'est transformé en une attraction dans les itinéraires touristiques ${ }^{49}$. Les municipalités s'attendent à ce que cette politique ait un impact sur l'économie locale. L'une des mesures déjà mises en œuvre est la transformation des répétitions (un contexte privé de perfectionnement et d'apprentissage pour l'individu comme pour l'ensemble) en spectacles ouverts au public, et en particulier aux touristes. Les ensembles souscrivent à cette initiative car ils n'ont pas la capacité de négocier. Mais lors des rencontres publiques, ils ont exprimé leur inquiétude quant à cette mesure : ils craignent d'être bloqués dans leur localité et de ne plus bénéficier du soutien nécessaire pour les tournées.

Le changement survenu au sein des familles, où les plus jeunes sont devenus un facteur de transmission et de motivation pour chanter le cante, a été souligné par tous. La citation qui ouvre notre article essaie d'en témoigner. Cette situation a été rapportée de façon répétée : des familles où des citadins d'âge moyen (entre 30 et 50 ans), qui ne s'intéressaient pas à cette pratique musicale, ont redécouvert le cante à travers leurs jeunes enfants et pré-adolescents l'ayant appris à l'école. Cette transformation apparaît aussi dans la redéfinition du chant survenue ces dernières années dans la vie sociale des jeunes urbains (période pré-candidature et post-inscription dans la liste de l'UNESCO). Savoir chanter à la façon de l'Alentejo est pour les jeunes une occasion de participer à la vie sociale de leurs pairs et une promesse d'entrée dans le monde du spectacle et des médias. Le nombre d'ensembles, formellement institués ou non, a 
augmenté, et la classe d'âge de ceux qui détiennent cette connaissance s'est élargie. Les projets d'enseignement du cante aux enfants dans les écoles publiques et/ou dans des groupes organisés de manière officielle se sont également développés. Les membres les plus âgés des ensembles reconnaissent le nouvel intérêt dont jouit le cante alentejano, mais voient avec appréhension la visibilité accrue d'un nombre restreint de mestres et d'enseignants, qu'ils jugent dangereuse, car pouvant entraîner la perte de ce qu'ils considèrent être des différences régionales.

Cette étude a mis en évidence que le processus d'inscription du cante alentejano sur la liste du Patrimoine Immatériel de l'Humanité de l'UNESCO a généré des transformations qui ont eu un impact sur la façon dont les Alentejanos eux-mêmes perçoivent leur culture et leurs traditions musicales. Des discours non-nostalgiques sur le cante alentejano ont émergé, centrés sur l'avenir de cette pratique - c'est-à-dire, "vers où allons-nous", selon l'expression de Stuart Hall ${ }^{50}$-, l'espoir de nouvelles rencontres, expériences et occasions de participation à la vie sociale, à l'intérieur et à l'extérieur de l'aire géographique de l'Alentejo.

\section{BIBLIOGRAPHIE}

ANDERSON Benedict, Imagined Communities, London and New York, Verso, 2000.

AuClAir Elizabeth, FAIRCLOUGH Graham, "Living Between Past and Future: An Introduction to Heritage and Cultural Sustainability", AUCLAIR Elizabeth, FAIRCLOUGH Graham (dir.), Theory and Practice in Heritage and Sustainability, London et New York, Routledge, 2015, p. 1-22.

BARRIGA Maria José, «Baldão», CASTELO-BRANCo Salwa (dir.), Enciclopédia da Música em Portugal no Século XX, Lisboa, Círculo de Leitores, 2010, p. 101-102.

BARRIGA Maria José, «Repentismo e folclorização no Baixo Alentejo: o cante ao baldão», CASTELOBRANCo Salwa, BRANCo Jorge Freitas (dir.), Vozes do Povo A Folclorização em Portugal, Lisboa, Celta Editora, 2003, p. 275-280.

BARRIGA Maria José, Cante ao baldão uma prática de desafio no Alentejo, Lisboa, Edições Colibri, 2000.

CABEÇA Sónia Moreira, SANTOS José Rodrigues dos, «A mulher no Cante Alentejano», Proceedings of the International Conference in Oral Tradition, Ourense, Concelho de Ourense, Vol. II, 2010, p. 31-38.

CABEÇA Sónia Moreira, Estrutura e processo de formação das formas culturais: o caso do Cante Alentejano, Évora, Universiade de Évora, 2016.

CARTAGENo António, «O Alentejo e os outros mundos no canto alentejano», Arquivo de Beja, série III, vol. VII, Beja, 1998, p. 387-392.

Castelo-Branco Salwa e Branco Jorge Freitas (dir.), Vozes do Povo A Folclorização em Portugal, Lisboa, Celta, 2003.

Castelo-Branco Salwa, "Some aspects of the 'Cante' Tradition of Cuba: A Town in Southern Alentejo, Portugal”, Livro de Homenagem a Macário Santiago Kastner, Lisboa, Fundação Calouste Gulbenkian, 1992, p. 546-561. 
CAStelo-BRAnco Salwa, "The Politics and Aesthetics of Multipart Singing in Southern Portugal", AHMEDAJA Ardian and HAID Gerlinde (eds.), European Voices: Multipart Singing in the Balkans, Vienna, Bohlau Verlag, 2008, p. 15-37.

CASTELO-BRANCo Salwa, «Musical Traditions of Portugal», BAUMAnN Max Peter (ed.), The World's Musical Traditions 9, Washington, International Institute for Traditional Music (CD), 1994, p. 24-34. CAstelo-Branco Salwa, «Sons da Memória : Os Registos Sonoros de Armando Leça no Alentejo / Sounds of Memory: Armando Leça's Sound Recordings from Alentejo», PESTANA Maria do Rosário (ed.), The Alentejo: Voices and Aesthetics in 1939/40. A critical edition of Armando Leça's sound recordings, Vila-Verde, Tradisom, 2014, p. 94-101.

Castelo-branco Salwa, Voix du Portugal, Paris, Cité de la Musique/Actes Sud, 1997.

CORTEZ Maria Rita Ortigão Pinto, Cancioneiro de Serpa, Serpa, Câmara Municipal, 1994.

DeLGADo Manuel Joaquim, A etnografia e o folclore do Baixo Alentejo, Lisboa, [s/n], 1958.

DELGADo Manuel Joaquim, Subsídio para o Cancioneiro Popular do Baixo Alentejo, vol. 1, no. 2, Lisboa, [s/n], 1955.

GRAÇA Fernando Lopes, «Apontamento sobre a canção alentejana», A Música Portuguesa e os Seus Problemas, vol. III, Lisboa, Cosmos, 1973, p. 175-183.

HALL Stuart, “Introduction : Who needs 'Identity'?, HALl Stuart, GAY Paul du (dir.), Questions of Cultural Identity, London, Sage, 1996, p. 1-17.

HANDLER Richard, Nationalism and the Politics of Culture in Quebec, Madison, The Wisconsin University Press, 1988.

ISNART Cyril et SANTOS José Rodrigues dos, « Le mestre et son cours : figure et institution de la transmission patrimoniale du chant dans le sud du Portugal », ADELL Nicolas et POURCHER Yves (dir.), Transmettre, quel(s) patrimoine(s) ?, Paris, Michel Houdiard Éditeur, 2011, p. 167-180.

KIRSHENBLATT-GIMBLETT Barbara, "From Ethnology to Heritage: The Role of the Museum", SIEF Keynote, Marseille, April 28, 2004, p. 1, URL: http://www.nyu.wdu/classes/bkg/web/SIEF.pdf (consulté le 21 octobre 2018).

KIRSHENBLATT-GIMBLETT Barbara, "Intangible Heritage as Metacultural Production", Museum, vol. 56/1-2, no. 221-222, 2004, p. 53.

KIRSHENBLATT-GIMBLETT Barbara, Destination Culture: Tourism, Museums, and Heritage, Berkeley and Los Angeles, University of California Press, 1996.

LIMA Paulo, «Dossier UNESCO do Cante Alentejano in Dossier UNESCO do Cante Alentejano », A Tradição, Nova série, vol. 0, 2015.

MARVÃo António, Cancioneiro Alentejano: corais majestosos, coreográficos e religiosos do Baixo Alentejo, Braga, Tip. Ed. Franciscana, 1955.

MARVÃo António, Estudos sobre o cante alentejano, Lisboa, Inatel, 1997 ; NAZARÉ João Ranita da, Prolegomenes à l'ethnosociologie de la musique, Paris, Fundação Calouste Gulbenkian, Centre Culturel Portugais, 1984.

NAZARÉ João Ranita da, Momentos Vocais do Baixo Alentejo: cancioneiro da Tradição Oral, Lisboa, Imprensa Nacional Casa da Moeda, 1986.

OLIVEIRA Ernesto Veiga de, Instrumentos Populares Portugueses, Lisboa, CAIMAV, Centro de Apoio à Investigação pelos Meios Audio-visuais, 1986. 
PESTANA Maria do Rosário e RIBEIRo Jorge Castro, Folclore e Folclorização no Montijo: trânsitos e encontros da música e da dança, Lisboa, Colibri, 2013.

Pestana Maria do Rosário, OliveIRA Luísa Tiago de (dir.), Cantar no Alentejo: A Terra, o passado e o presente, Lisboa, Estremoz Editora, 2017.

Pestana Maria do Rosário, The Alentejo: Voices and Aesthetics in 1939/40. A Critical Edition of Armando Leça's Sound Recordings, Vila Verde, Tradisom, 2014.

PetTAn Svanibor, Titon Jeff Todd (dir.), The Oxford Handbook of Applied Ethnomusicology, Oxford et New York, Oxford University Press, 2015.

SANTos Vítor, Cancioneiro Alentejano, Lisboa, Grémio Alentejano Artigos, 1938.

\section{NOTES}

1. Cette étude a été réalisée dans le cadre du projet "Our music, our world: Musical associations, wind bands, and local communities (1880-2018)", PTDC/CPC-MMU/5720/2014, financée par la Fondation pour la Science et la Technologie, POCI-01-0145-FEDER-016814 et coordonnée par Maria do Rosário Pestana.

2. «Eu tomei o gosto pelo cante com o meu pai, em Serpa, da taberna em frente da casa... e a arrepiar... aquela coisa maravilhosa deles... portanto, a mim tocou-me. 0 meu filho mais velho tem 25 anos e não apanhou esta onda do ensino do cante [alentejano] nas Escolas. Mas tem a mãe com um gosto muito grande por cantar e sempre que vou para Serpa, para o Algarve... vou sempre cantando e ele mandava-me calar: Oh, Mãe! "Que piroso! Que parolice!". O outro meu filho de 21 anos, a mesma conversa: - Oh mãe cale-se, lá está a mãe com as cantorias... Agora, este [refere-se ao terceiro filho, o mais novo] já apanhou esta onda. Teve quatro anos de cante [alentejano] na escola. o facto de os pares, os colegas cantarem, o facto de serem grupos de jovens na puberdade, faz com que o meu filho me pergunte: - Mãe como é que era aquela canção que a mãe canta? A ver se eu canto, porque quando se reúne com os colegas, depois, não sabe. Portanto, isto tem muita influência. Os pais, os grupos, a escola, o sentirem-se pertença de um grupo que canta, no fundo é isto que vocês [vira-se para os cantadores que estão na mesa redonda à sua volta] gostam todos quando vão cantar nos grupos. É a pertença no grupo. É a identidade: o encontro no outro. E isto é uma coisa maravilhosa que o cante alentejano nos faz sentir.» (Teresa Almeida, Centro UNESCO de Beja, 2018)

3. L'expression "le patrimoine c'est nous" est également utilisée par Daniel Fabre dans son ouvrage Émotions patrimoniales (2013, p. 17), pour désigner le tournant patrimonial des sociétés européennes (note des coordinateurs).

4. Ce mouvement avait débuté avant 2014, sous l'action d'associations locales et municipales, par une démarche centrée sur l'enseignement du cante alentejano dans les écoles et avec l'apparition d'ensembles de jeunes comme «Os Bubedanas» de Beja, «Os Mainantes» de Pias, «Os Seca Adegas» de Vila Ruiva ou encore les «Bafos de Baco» de Cuba.

5. "[...] metacultural operations that extend museological values and methods [...] to living persons". KIRSHENBLATT-GIMBLETT Barbara, "From Ethnology to Heritage: The Role of the Museum", SIEF Keynote, Marseille, April 28, 2004, p. 1, URL: nyu.edu/classes/bkg/web/SIEF.pdf (consulté le 21 octobre 2018).

6. "This means according value to the 'carriers' and 'transmitters' of traditions, as well as to their habitus and habitat [...] to sustain the whole system as a living entity." KIRSHENBLATT-GIMBLETT Barbara, "Intangible Heritage as Metacultural Production", Museum, vol. 56/1-2, no. 221-222, 2004, p. 53.

7. Auclair Elizabeth, Fairclough Graham, "Living Between Past and Future: An Introduction to Heritage and Cultural Sustainability", AUCLAIR Elizabeth, FAIRCLOUGH Graham (eds.), Theory and 
Practice in Heritage and Sustainability, London et New York, Routledge, 2015, p. 1-22. Les auteurs analysent les manières dont le patrimoine s'inscrit dans les processus de développement durable. 8. PetTAn Svanibor, Titon Jeff Todd (eds.), The Oxford Handbook of Applied Ethnomusicology, Oxford et New York, Oxford University Press, 2015. Les auteurs de ce livre soutiennent une pratique ethnomusicologique guidée par des principes éthiques de responsabilité sociale, en s'engageant en faveur des communautés étudiées et en cherchant à répondre à leurs besoins.

9. Pestana Maria do Rosário, Oliveira Luísa Tiago de (dir.), Cantar no Alentejo: A Terra, o passado e o presente, Lisboa, Estremoz Editora, 2017.

10. BARRIGa Maria José, Cante ao baldão uma prática de desafio no Alentejo, Lisboa, Edições Colibri, 2000 ; BARRIGA Maria José, «Repentismo e folclorização no Baixo Alentejo: o cante ao baldão», Castelo-branco Salwa, Branco Jorge Freitas (dir.), Vozes do Povo A Folclorização em Portugal, Lisboa, Celta Editora, 2003, p. 275-280 ; BARRIGA Maria José, «Baldão», C ASTElo-BRANCo Salwa (dir.), Enciclopédia da Música em Portugal no Século XX, Lisboa, Círculo de Leitores, 2010, p. 101-102; BARRIGA Maria José, «Cante ao baldão: uma prática de desafio no Alentejo entre 1980 e a actualidade», Pestana, Oliveira (dir.), Cantar no Alentejo, p. 119-132.

11. Pestana Maria do Rosário, The Alentejo: Voices and Aesthetics in 1939/40. A Critical Edition of Armando Leça's Sound Recordings, Vila Verde, Tradisom, 2014 ; PeSTANA Maria do Rosário, «Alentejo, visibilidade e ocultação: scriptualização e institucionalização de práticas musicais rurais», Pestana, Oliveira (dir.), Cantar no Alentejo, p. 133-149.

12. Cante dérive de « canto » [chant].

13. «O ponto e o alto têm um papel de liderança no canto. Qualquer um deles ou, em alternativa, outro portador de tradição, lidera o grupo, ensinando os cantadores, selecionando e transmitindo o repertório, conduzindo os ensaios e exibições. Este papel de mestre do coro foi recentemente formalizado, recebendo o reconhecimento público e o título de mestre». LIMA Paulo, «Dossier UNESCO do Cante Alentejano in Dossier UNESCO do Cante Alentejano ", A Tradição, Nova série, vol. 0, 2015, p. 18, notre traduction.

14. Pour mieux connaître la pratique du cante alentejano, on pourra consulter les travaux suivants : CABEÇA Sónia Moreira, Estrutura e processo de formação das formas culturais : o caso do Cante Alentejano, Évora, Universidade de Évora, 2016 ; CARTAGEno António, «O Alentejo e os outros mundos no canto alentejano", Arquivo de Beja, série III, vol. VII, Beja, 1998, p. 387-392 ; CASTELOBRANCO Salwa, "Some aspects of the 'Cante' Tradition of Cuba: A Town in Southern Alentejo, Portugal”, Livro de Homenagem a Macário Santiago Kastner, Lisboa, Fundação Calouste Gulbenkian, 1992, p. 546-561 ; CASTELO-BRANCO Salwa, «Musical Traditions of Portugal», BAumanN Max Peter (ed.), The World's Musical Traditions 9, Washington, International Institute for Traditional Music (CD), 1994, p. 24-34 ; CASTELo-BRAnCo Salwa, Voix du Portugal, Paris, Cité de la Musique/Actes Sud, 1997 ; CAStelo-BRanco Salwa, "The Politics and Aesthetics of Multipart Singing in Southern Portugal", AHMEDAJA Ardian and HAID Gerlinde (eds.), European Voices: Multipart Singing in the Balkans, Vienna, Bohlau Verlag, 2008, p. 15-37; CASTElo-Branco Salwa, «Sons da Memória: Os Registos Sonoros de Armando Leça no Alentejo / Sounds of Memory: Armando Leça's Sound Recordings from Alentejo», PestanA Maria do Rosário (ed.), The Alentejo: Voices and Aesthetics in 1939/40. A critical edition of Armando Leça's sound recordings, Vila-Verde, Tradisom, 2014, p. 94-101; Castelo-branco Salwa, «Para o estudo dos Patrimónios Sonoros do Alentejo», Pestana, Oliveira (dir.), Cantar no Alentejo, p. 1-11; IsNART Cyril et SANTOS José Rodrigues dos, «Le mestre et son cours : figure et institution de la transmission patrimoniale du chant dans le sud du Portugal ", ADELl Nicolas et POURCHER Yves (dir.), Transmettre, quel(s) patrimoine(s) ?, Paris, Michel Houdiard Éditeur, 2011, p. 167-180 ; CORTEZ Maria Rita Ortigão Pinto, Cancioneiro de Serpa, Serpa, Câmara Municipal, 1994 ; DELGADo Manuel Joaquim, Subsídio para o Cancioneiro Popular do Baixo Alentejo, vol. 1, no. 2, Lisboa, [s/n], 1955 ; DelgAdo Manuel Joaquim, A etnografia e o folclore do Baixo Alentejo, Lisboa, [s/n], 1958 ; GRAÇA Fernando Lopes, «Apontamento sobre a canção alentejana», A Música 
Portuguesa e os Seus Problemas, vol. III, Lisboa, Cosmos, 1973, p. 175-183; MARVÃo António, Cancioneiro Alentejano: corais majestosos, coreográficos e religiosos do Baixo Alentejo, Braga, Tip. Ed. Franciscana, 1955 ; MARVÃo António, Estudos sobre o cante alentejano, Lisboa, Inatel, 1997 ; NAZARÉ João Ranita da, Prolegomenes à l'ethnosociologie de la musique, Paris, Fundação Calouste Gulbenkian, Centre Culturel Portugais, 1984; NAZARÉ João Ranita da, Momentos Vocais do Baixo Alentejo: cancioneiro da Tradição Oral, Lisboa, Imprensa Nacional Casa da Moeda, 1986 ; OLIVEIRA Ernesto Veiga de, Instrumentos Populares Portugueses, Lisboa, CAIMAV, Centro de Apoio à Investigação pelos Meios Audio-visuais, 1986 ; SANTos Vítor, Cancioneiro Alentejano, Lisboa, Grémio Alentejano Artigos, 1938.

15. CASTElo-BRAnCo, "Some Aspects of the 'Cante' Tradition of Cuba”, p. 552.

16. CABEÇA Sónia Moreira, Estrutura e processo de formação das formas culturais: o caso do Cante Alentejano, Évora, Universiade de Évora, 2016.

17. Recensement 2011, Résultats préliminaires. Région Alentejo. Cf. : censos.ine.pt/xportal/ xmain?

xpid=CENSOS\&xpgid=ine_censos_publicacao_det\&menuBOUI=13707294\&contexto=pu\&PUBLICACOESpub_boui=377789\&PUBLICACOESn (consulté le 20 août 2019). Instituto Nacional de Estatística, Censos 2011 Resultados Definitivos Região Alentejo, Lisboa, Instituto Nacional de Estatística, I.P., 2011.

18. BAPTISTA Fernando Oliveira, «Alentejo, Terra, trabalho, património», PESTANA Maria do Rosário, oliveIRA Luísa Tiago de (dir.), Cantar no Alentejo: A Terra, o passado e o presente, Lisboa, Estremoz Editora, 2017, p. 35. Fernando de Oliveira Baptista - ministre de l'Agriculture des gouvernements qui ont légalisé la Réforme Agraire dans les années suivant le rétablissement de la démocratie au Portugal - analyse les transformations de l'utilisation des terres agricoles de l'Alentejo depuis la fin du XIX ${ }^{\mathrm{e}}$ siècle.

19. BAPTISTA, «Alentejo, Terra, trabalho, património», p. 35.

20. HANDLER Richard, Nationalism and the Politics of Culture in Quebec, Madison, The Wisconsin University Press, 1988. L'anthropologue Richard Handler analyse le processus « d'objectivation culturelle» au Québec qui comprend la sélection d'éléments, leur décontextualisation et l'attribution d'un nouveau sens culturel.

21. SIMÕES Dulce, "A turistificação do Cante Alentejano como estratégia de "desenvolvimento sustentável": discursos políticos e práticas da cultura», Pestana, OliveIRA (dir.), Cantar no Alentejo, p. 59-88.

22. CABEÇA Sónia Moreira, SANTOS José Rodrigues dos, «A mulher no Cante Alentejano», Proceedings of the International Conference in Oral Tradition, Ourense, Concelho de Ourense, Vol. II, 2010, p. 31-38.

23. BARRIGA, Cante ao baldão uma prática de desafio.

24. Les 81 enregistrements sonores collectés en Alentejo ont été édités pour la première fois en 2014, avec les notes de terrain et les photographies réalisées par Armando Leça; PESTANA, The Alentejo: Voices and Aesthetics in 1939/40.

25. oliveira Luísa Tiago, "O Alentejo de Michel Giacometti», Pestana, Oliveira (dir.), Cantar no Alentejo, p. 151-182.

26. CAstelo-Branco, "The Politics and Aesthetics of Multipart Singing in Southern Portugal", p. $15-37$.

27. «O número de grupos masculinos foi sempre superior ao número de grupos femininos ; no entanto, a formação de grupos femininos mantém-se em crescimento desde a década de 1980. 0 reforço das mulheres nos grupos corais reclama 'uma visão holística do património cultural imaterial e uma nova compreensão da memória coletiva futura'». CABEÇA, SANTOS, «A mulher no Cante Alentejano», p. 14.

28. "Municipalities replaced the governmment in providing financial and logistical support (rehearsal space, transportation, etc.)." CASTELO-BRANCO, "The Politics and Aesthetics of Multipart Singing in Southern Portugal", p. 23. 
29. CASTELO-BRANCO Salwa e BRANCo Jorge Freitas, «Folclorização em Portugal: uma perspectiva», CASTElo-Branco Salwa e Branco Jorge Freitas (dir.), Vozes do Povo A Folclorização em Portugal, Lisboa, Celta, 2003, p. 1-24 ; PESTANA Maria do Rosário e RIBEIRo Jorge Castro, Folclore e Folclorização no Montijo: trânsitos e encontros da música e da dança, Lisboa, Colibri, 2013.

30. Castelo-branco Salwa, "The Politics and Aesthetics of Multipart Singing in Southern Portugal”, p. 24. ANDERSON Benedict, Imagined Communities, London and New York, Verso, 2000.

31. Le dossier de candidature a été publié par la mairie de Serpa, A Tradição, Nova série, 0, 2015, et peut être téléchargé sur le site de la Municipalité : cm-serpa.pt/casadocante/pdf/ A_TRADICAO_0.pdf (consulté le 20 août 2019).

32. Pestana, Oliveira (dir.), Cantar no Alentejo, p. 238-239.

33. «Estimulou o interesse dos jovens por este modo expressivo, levando à formação de grupos corais constituídos exclusivamente por jovens, à sua integração em alguns grupos existentes, à formação de pequenos grupos constituídos por cantadores acompanhados pela viola campaniça e à configuração de novos idiomas musicais que misturam o cante com diversos estilos de música popular.», CASTELO-BRANCO Salwa, «Para o estudo dos Patrimónios Sonoros do Alentejo», Pestana, Oliveira (dir.), Cantar no Alentejo, p. 1-11.

34. Ce recensement a été réalisé dans le cadre du projet "Music in-Between: the 'Orfeonismo' Movement and Choral Singing in Portugal (1880-2012)", PTDC/EAT-MMU/117788/2010, financé par la Fondation pour la Science et la Technologie, Compete et QREN et coordonné à partir de l'université d'Aveiro par Maria do Rosário Pestana.

35. «O principal aspecto que diferencia estes artistas dos grupos corais (tanto da antiga como da nova geração) [...] é a música é a sua actividade profissional e são concorrentes entre si no que toca aos públicos, às editoras, às rádios, etc.», nous traduisons. MARECO Susana, «A Nova Geração do Cante e as Manifestações sobre o Cante Alentejano», Pestana, Oliveira (dir.), Cantar no Alentejo, p. 89-118.

36. MOVIMENTO DEMOCRÁTICO DAS MULHERES, O Cante no Feminino As vozes das mulheres no Património Cultural Imaterial, Lisboa, MDM, 2016.

37. «O balanço que fazemos, passados dez anos, é que gostaríamos que as crianças que têm interesse em aprender mais, mas que são ainda muito jovens para integrar os grupos, pudessem continuar a aprender. Pensamos em oferecer aqui na Casa do Cante aulas aos sábados, com o contributo dos grupos corais [...]. É preciso acontecer mais qualquer coisa [do que o Cante no primeiro ciclo] e para acontecer mais qualquer coisa é fundamental que sejam vocês [dirige-se aos cantadores que estão na plateia] a transmitir esse conhecimento. Pois eu sei que há algumas críticas que dizem: - 'o que se está a fazer com o Cante nas escolas é tudo igual'. Porque nós temos em Serpa o cantar de Pias, o cantar de Vila Nova... Cada terra e cada grupo tem as suas características. Claro que só quem integra os grupos aprenderá! Mas é importante que as crianças em quem estamos a depositar essa semente e que a querem fazer crescer, tenham esse apoio. Queria fazer aqui um compromisso entre os grupos corais nessa transmissão.» Odete Borralho, Casa do Cante de Serpa, 2018.

38. "A escola do Telheiro [deve ter] um Cante, a escola de Reguengos terá outro, a de Portel terá outro, a Amareleja terá outro. Não se pode estandardizar, como a Matemática!», PeSTANA, OliveIRA (dir.), Cantar no Alentejo, p. 203.

39. Ibid, p. 230.

40. «Não tenho na minha ideia ir para uma escola e fazer 30 ou 40 cantadores, o que se pretende é sensibilizar e informar as crianças de que existe este património, esta cultura, esta identidade que faz parte da nossa terra. É importante elas saberem, independentemente de o seguirem ou não. E é isso que nos move. Se hoje nós damos graças por ter aprendido com os grupos que existiram há setenta/oitenta anos, também queremos que, daqui a cinquenta anos, os mais novos possam dizer de nós : estes senhores cantam há cinquenta anos. Se não for assim, se não houver sempre esta transmissão de conhecimento para as crianças mais novas, vai chegar um dia em que isto vai morrer, como uma fonte.» Ibid, p. 207. 
41. «Na década de 1990 [...] quando cantava no cortejo, com 13 anos, tinha grupos de amigos a assobiar-me quando passava a cantar. 0 Cante era proibido nos cafés, nos restaurantes [...] houve ali uma fase que quase era foleiro cantar porque estava conotado com pessoas ligadas ao álcool. Com a UNESCO começou-se a falar do Cante com um interesse cultural. [...]As mentalidades foram-se alterando e hoje em dia onde era proibido cantar, pedem para se cantar, temos uma realidade distinta.» Carlos Arruda, Casa do Cante de Serpa, 2018.

42. «O Património Cultural e Imaterial da Humanidade veio trazer outra projeção ao Cante. Os jovens aprenderam a gostar do Cante e os adultos também. [...] Antes, a maioria das pessoas achavam aquilo piroso, achavam aquilo fatela, não gostavam.» José Soares, Casa do Cante de Serpa, 2018.

43. «O Cante tem hoje uma importância que não lhe era dada há dez anos. Nessa altura, quando integrei o grupo, entrar num coral de "gente velha" era como parar no tempo, não poder ir jogar futebol, não estar com os amigos, não estar onde estavam todos: Na patinagem, no futebol, no andebol e tudo mais. Estar isolado com cinquenta, vinte, trinta homens a cantar à alentejana era, assim, um escândalo que fazia do António [irmão] e de mim dois meninos retrógrados [...] felizmente que houve a candidatura a Património Imaterial da Humanidade [...] e que fomos pioneiros na sua salvaguarda.» PESTANA, OLIVEIRA (dir.), Cantar no Alentejo, p. 201.

44. «Somos um grupo do concelho de Ourique e não somos chamados a lado nenhum. Depois vem o presidente da câmara no dia que fazemos lá [na localidade sede do grupo] a festa e diz: "Temos de preservar o cante alentejano!" E eu pergunto: de que maneira?» José Rita, Casa do Povo de S. Martinho das Amoreiras, commune de Odemira, 2018.

45. «Uma forma de ajudar as nossas aldeias e agora com o turismo, é o grupo não sair tanto, e atuar mais na sua aldeia e receber as pessoas de fora. 0 grupo tem que representar a terra e hoje em dia temos que repensar isto das saídas. Gostar de receber os amigos e as pessoas de fora a cantar é importante.» Paulo Colaço, Centre Unesco de Beja, 2018.

46. «Porque nós também cantávamos, cantavam os homens e as mulheres nesse tempo. Eu tenho 69 anos e eu trabalhei no campo toda a minha vida e nós as mulheres cantávamos ceifando, apanhando grãos... Eu sei que era assim! [...]. Talvez esteja enraizado na maior parte dos homens que as mulheres não cantavam, principalmente aqui em Serpa. As mulheres não cantavam nas tabernas, mas cantavam nos trabalhos e em casa!» Ana Rita Nogueira Esparteiro Casa do Cante de Serpa, 2018.

47. «Nós no Grupo Coral Os Ceifeiros de Serpa temos duas jovens, uma com 10 anos e outra de 15 e elas não falham, gostam mesmo de andar lá. [...] 0 avô delas cantava no grupo de Pias, era um bom elemento, e elas querem seguir os passos do avô. Dizem mesmo que gostam que o avô, esteja onde estiver, as veja a andar lá. Têm orgulho.» Guiomar Rita, Casa do Cante de Serpa, 2018.

48. «O que é preciso fazer para que todos os grupos pudessem correr esse terreno fora do cante, é aquilo precisamente que a Casa do Cante de Serpa começou a fazer há tempos e o Centro UNESCO de Beja. E o que é que fizeram essas entidades, estão a criar uma plataforma, um site, que possa ter todos os grupos lá. 0 problema é quando essas entidades pedem aos grupos para lhes mandarem informação e os grupos não têm capacidade para o fazer. Pedem aos grupos: atualizem os vossos dados nesta página! [on-line]. Mas os grupos não têm capacidade para o fazer. São trabalhadores rurais na maioria dos casos. Esquecemos dessa parte. A UNESCO deu o selo ao cante, quer defender o cante amador, mas depois esquece-se que essas pessoas que não estão aptas para dar essas respostas. Então era necessário ajudar essas pessoas diretamente. Quando eu digo diretamente, é lá nos ensaios deles.» Pedro Mestre, Casa do Povo de S. Martinho das Amoreiras, commune de Odemira, 2018.

49. KirshenblatT-GIMBLETT Barbara, Destination Culture: Tourism, Museums, and Heritage, Berkeley and Los Angeles, University of California Press, 1996.

50. HaLl Stuart, “Introduction: Who needs “Identity’?, HaLl Stuart, GAY Paul du (dir.), Questions of Cultural Identity, London, Sage, 1996, p. 1-17. 


\section{RÉSUMÉS}

Le cante alentejano, inscrit sur la Liste du Patrimoine Culturel Immatériel de l'UNESCO en 2014, est un chant polyphonique originaire de la région de l'Alentejo, au sud du Portugal, et qui se caractérise par la prédominance de trois rôles (ponto soliste, alto soliste et baixos ou chorale). Cette inscription a engendré l'émergence de nouveaux ensembles musicaux, stimulé l'activité des musiciens et dynamisé des politiques culturelles locales de sauvegarde des traditions musicales rurales, ainsi que l'économie liée au tourisme. Nous analysons ce contexte « post-inscription sur la liste de l'UNESCO " selon les tensions créées autour de polarités: apprentissage scolaire vs pratique au sein des ensembles locaux; chanteurs plus âgés vs jeune génération; ensembles traditionnels vs projets artistiques contemporains; politiques culturelles locales vs attentes et besoins des ensembles. Si l'on considère que le Patrimoine Culturel Immatériel se tisse et se transmet à travers des interactions et des échanges entre des personnes qui partagent une même connaissance, dans quelle mesure la reconnaissance de l'UNESCO affecte-t-elle les pratiques musicales locales? Comment les protagonistes du cante alentejano conçoivent-ils cette inscription? De quelle façon des ensembles vocaux constitués depuis des décennies à la faveur de processus d'«objectification culturelle» font-ils face à ces nouvelles transformations? Notre recherche est fondée sur un travail de terrain mené auprès de différents ensembles et musiciens, notamment à l'occasion de rencontres publiques organisées avec les acteurs de la musique et de sa revitalisation. L'étude révèle qu'au cours des dix dernières années (timidement pendant la période de préparation de la candidature, puis plus fortement après la reconnaissance de l'UNESCO), les discours et préoccupations qui ont émergé de la pratique du cante alentejano ont été principalement centrés sur l'espoir de disposer de nouvelles opportunités de participation à la vie sociale contemporaine, à l'intérieur comme à l'extérieur de l'aire géographique de l'Alentejo.

Cante alentejano, inscribed on the UNESCO List of Intangible Cultural Heritage in 2014, is a genre of polyphonic singing from the region of Alentejo in southern Portugal, characterised by the predominance of three roles (ponto soloist, alto soloist and baixos or chorus). Its inscription on the UNESCO List has led to the emergence of new musical ensembles, stimulated musician's activity, brought new impetus to local cultural policy on the safeguarding of rural musical traditions, and invigorated the tourism-related economy. We analyse this "post-inscription on the UNESCO list" context according to the tensions created around a number of polarities: learning at school vs practice with local ensembles; older singers vs the young generation; traditional ensembles vs modern-day artistic projects; local cultural policies vs ensembles' needs and expectations. If we consider that Intangible Cultural Heritage is woven and passed on through interactions and exchanges between people sharing a common body of knowledge, then to what extent does recognition by UNESCO affect local musical practices? How do the protagonists of cante alentejano perceive this inscription? How do vocal ensembles formed decades ago, that have worked in favour of "cultural objectification", respond to these new transformations? Our research is based on fieldwork carried out with different ensembles of musicians, notably through public gatherings organised with the stakeholders in the music and its revitalisation. The study shows that over the past ten years (mildly during the application preparation process, and more markedly since recognition by UNESCO), the discourse and concerns that have emerged from the cante alentejano practice have primarily centred around the hope of gaining new ways of participating in the social life of today, both within and outside the geographic area of Alentejo. 


\section{INDEX}

Keywords : cante alentejano, intangible cultural heritage, heritagisation, polyphonic singing, Portugal (region)

Mots-clés : cante alentejano, patrimoine culturel immatériel, patrimonialisation, chant polyphonique, Portugal (région)

\section{AUTEURS}

\section{MARIA DO ROSÁRIO PESTANA}

Maria do Rosário Pestana est docteure en Ethnomusicologie de la Faculté de Sciences Sociales et Humaines de l'Université Nova de Lisbonne, et professeure associée à l'Université d'Aveiro. Elle est membre de l'Institut d'Ethnomusicologie, Centre d'études en musique et danse (INET-md). Elle a développé des recherches de terrain et d'archives au Portugal, en France et en Suisse, qui ont abouti à des publications sur le folklore, la musique et l'émigration, les communautés musicales, la musique et les mouvements sociaux. Ses publications récentes comprennent la coordination des livres : Industries de la musique et des archives sonores au Portugal : pratiques, contextes, patrimoine (2013) ; The Alentejo: Voices and Aesthetics in 1939/40. A Critical Edition of Armando Leça's Sound Recordings (2014); Vozes ao Alto: Chanter en chœur au Portugal (2014); et Chanter dans l'Alentejo : la Terre, le passé et le présent (2017). Elle dirige deux projets de recherche financés par la Fondation pour la Science et la Technologie au Portugal : l'un centré sur les associations musicales, l'autre sur le postfolklorisme.

\section{MARIA JOSÉ BARRIGA}

Maria José Barriga est née à Beja (Sud du Portugal). Elle est diplômée en Clavecin du Conservatoire Royal de La Haye (Hollande, 1992), sous la direction du prof. Ton Koopman. Elle est claveciniste en orchestres et musique de chambre. Elle a réalisé des concerts au Portugal, en Espagne, en Hollande, en Allemagne et au Mexique. Elle est professeur de clavecin et de musique de chambre à l'Académie Sainte-Cécile et à l'Institut Grégorien de Lisbonne. Elle détient une Maîtrise en Ethnomusicologie de l'Université Nova de Lisboa, et mène en ce moment un Doctorat en Ethnomusicologie au sein de la même université. Ses recherches portent sur les chants traditionnels d'improvisation et les joutes chantées dans le Sud du Portugal. Elle a publié : Cante ao baldão - Uma prática de desafio no Alentejo (2003) aux éditions Colibri. 